\title{
Superposición de síndrome de Stevens Johnson/necrólisis epidérmica tóxica asociada con hipogammaglobulinemia en el periodo neonatal: informe de caso
}

\author{
Stevens Johnson/toxic epidermal necrolysis overlap syndrome associated \\ with hypogammaglobulinemia in the neonatal period: case report
}

\author{
Dra. Diana Guadalupe Ramírez-Vásquez, ${ }^{*}$ Dra. Orquídea Julián-García, ${ }^{\ddagger}$ \\ Dra. Lorena Ramírez-Cruz, ${ }^{\ddagger}$ Dra. Rocío Martínez-Canseco ${ }^{\ddagger}$ \\ * Jefa de Pediatría, alergóloga e inmunóloga. \\ ${ }^{\ddagger}$ Adscrita de Neonatología.
}

Hospital de la Niñez Oaxaqueña. Oaxaca, México.

\begin{abstract}
Citar como: Ramírez-Vásquez DG, Julian-García O, Ramírez-Cruz L, Martínez-Canseco R. Superposición de síndrome de Stevens Johnson/necrólisis epidérmica tóxica asociada con hipogammaglobulinemia en el periodo neonatal: informe de caso. Alergia Asma Inmunol Pediatr. 2021; 30 (2): 58-61. https://dx.doi.org/10.35366/101644
\end{abstract}

\section{RESUMEN}

La superposición de síndrome de Stevens Johnson (SSJ) con necrólisis epidérmica tóxica (NET) es una reacción farmacológica aguda, poco frecuente y asociada con una mortalidad hasta del $29 \%$. Según la clasificación ampliamente aceptada, tal sobreposición SSJ/NET se considera una manifestación extensa del SSJ, afectando entre el $10-30 \%$ de la superficie corporal, ocasionada principalmente por fármacos e infecciones y presentándose en forma de ampollas y erosiones. Reporte de caso: Se presenta el caso de un paciente masculino de un mes de edad, quien a los 12 días de vida presentó una dermatosis generalizada, con afección de mucosas caracterizadas por ámpulas y costras hemáticas con reporte histopatológico de dermatitis de interfaz vacuolar pauciinflamatoria linfocitaria perivascular superficial, compatible con necrólisis tóxica en la epidermis. Se estableció tratamiento con gammaglobulina humana al $10 \%$ a $2 \mathrm{~g} / \mathrm{kg} / \mathrm{dosis}$; sin embargo, posteriormente falleció por choque séptico secundario a Candida albicans. Conclusión: La sobreposición de SSJ/NET se considera extremadamente rara en el periodo neonatal, con pocos casos reportados, por lo que se debe considerar dentro del diagnóstico diferencial de las manifestaciones ampollosas y por esto realizar oportunamente la biopsia de piel.

\section{ABSTRACT}

The overlap of Stevens Johnson syndrome (SJS) and toxic epidermal necrolysis (TEN) is an acute, rare drug reaction associated to a mortality rate of up to $29 \%$. According to the widely accepted classification, the SJS/TEN overlap is considered an extensive manifestation of SJS, affecting between $10-30 \%$ of the body surface, presenting in the form of blisters and erosions caused mainly by drugs and infections. Case report: The case of a 1-month-old male patient is presented; at 12 days of age, he started showing generalized dermatosis with mucosal involvement characterized by blisters and blood crusts with a histopathological report of compatible superficial perivascular lymphocyte-pauciinflammatory vacuolar interface dermatitis along toxic epidermal necrolysis. Treatment using immunoglobulin was established at $2 \mathrm{~g} / \mathrm{kg} / \mathrm{do}$, however, he later died of septic shock secondary to Candida albicans. Conclusion: SJS/ TEN overlap is considered extremely rare in the neonatal period, it has few reported cases, so it should be considered within the differential diagnosis of bullous syndromes and therefore a skin biopsy shoud be performed in a timely manner.

Recibido: 16/11/2020. Aceptado: 17/09/2021. 
Palabras clave: Necrólisis epidérmica tóxica, síndrome de Stevens Johnson, farmacodermia, síndromes ampollosos, inmunodeficiencia.

\section{INTRODUCCIÓN}

La superposición del síndrome de Stevens Johnson con necrólisis epidérmica tóxica (SSJ/NET) es una enfermedad rara, aguda y potencialmente mortal, que se caracteriza por la aparición repentina de una necrosis generalizada que produce desprendimiento de la epidermis y afectación frecuente de las membranas mucosas. ${ }^{1}$ La incidencia anual se describe entre 1-4 casos/1,000,000 y se estima que el $20 \%$ del total de casos corresponden a pacientes en edades pediátricas. ${ }^{2}$ Está esencialmente ocasionada por una reacción adversa a fármacos, dentro de los cuales los más frecuentes son las sulfonamidas, mdeicamentos antiepilépticos y derivados de la penicilina. La infección es la segunda causa precipitante más común. Los principales gérmenes involucrados son: Mycoplasma pneumoniae, citomegalovirus, y herpes, entre otros. ${ }^{3}$ Dentro de la clasificación de reacciones alérgicas a medicinas, la sobreposición SSJ/ NET se encuentra en el tipo IVc, mediadas por linfocitos T citotóxicos a través de varias proteínas citotóxicas y citocinas como Fas ligando, perforina, granzima, factor de necrosis tumoral y granulisina -una proteína citolítica que se encuentra en linfocitos $\mathrm{T}$ y células $\mathrm{NK}^{4}$ - causando muerte

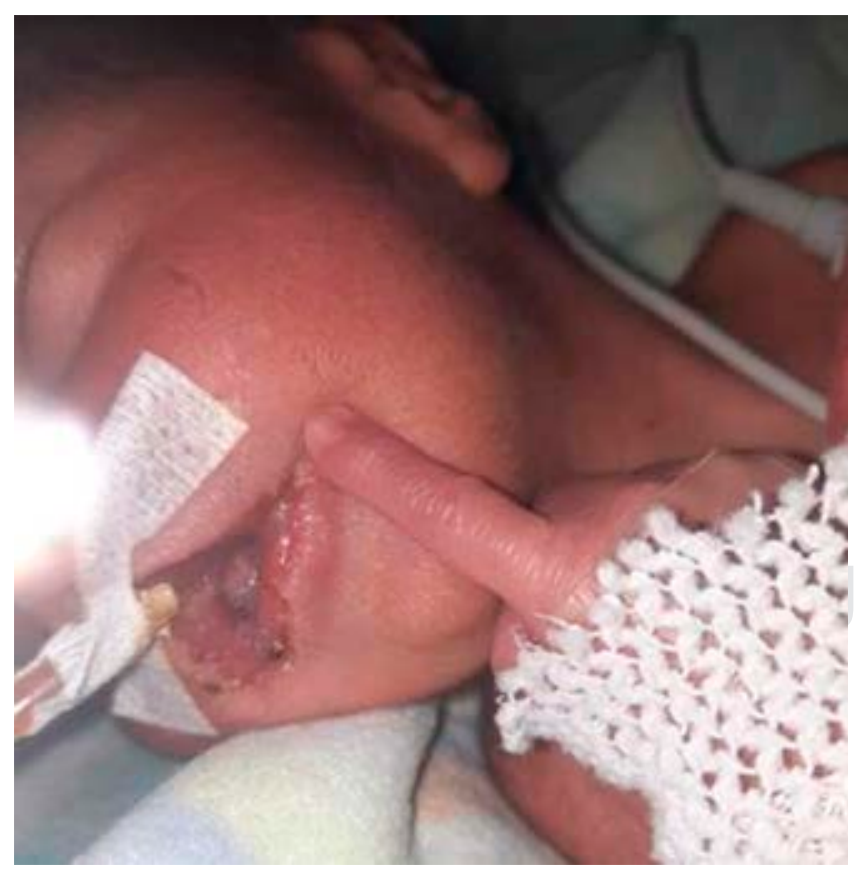

Figura 1: Mucosa oral afectada con descamación y ampollas.
Keywords: Toxic epidermal necrolysis, Stevens Johnson syndrome, pharmacoderma, bullous syndromes, immunodeficiency.

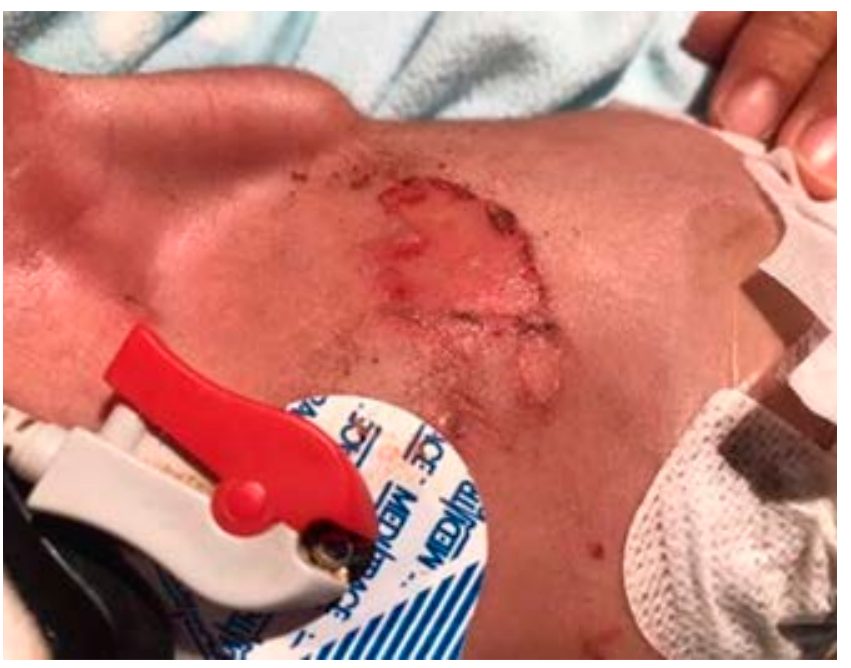

Figura 2: Desprendimiento de piel de abdomen y tórax de contorno mielicérico.

de queratinocitos mediados por CD4 o CD8. La reacción ocurre especialmente de cuatro a 28 días posterior al inicio del fármaco. ${ }^{5} \mathrm{Al}$ distinguir a los pacientes por gravedad, las tasas de mortalidad son del $12 \%$ para SSJ, 29\% para sobreposición SSJ/NET y $46 \%$ para NET.

En la práctica clínica se han utilizado varios tratamientos inmunosupresores o inmunomoduladores, incluidos los corticoides sistémicos, inmunoglobulina intravenosa, ciclosporina, plasmaféresis y anticuerpos monoclonales contra el factor de necrosis tumoral. ${ }^{6}$

\section{PRESENTACIÓN DEL CASO}

Se trata de un recién nacido sin endogamia ni consanguinidad, producto de la segunda gesta, obtenido por vía vaginal, de término y sin complicaciones, alimentado al seno materno con onfalorrexis a los cinco días. Inició su padecimiento a los cuatro días de vida extrauterina con vómitos de contenido gástrico y sangrado de tubo digestivo, por lo que se le ingresó al Servicio de Urgencias, con datos de deshidratación hipernatrémica, distermias y prolongación de tiempos de coagulación. Neurológicamente con hipoactividad, espasticidad y empuñamiento, por lo que se realizó tomografía axial computarizada (TAC) cerebral que reportó sangrado intraparenquimatoso. A su ingreso, se inició ampicilina y amikacina por sospecha de sepsis neonatal. A los 11 días de su ingreso, se dio la aparición de 


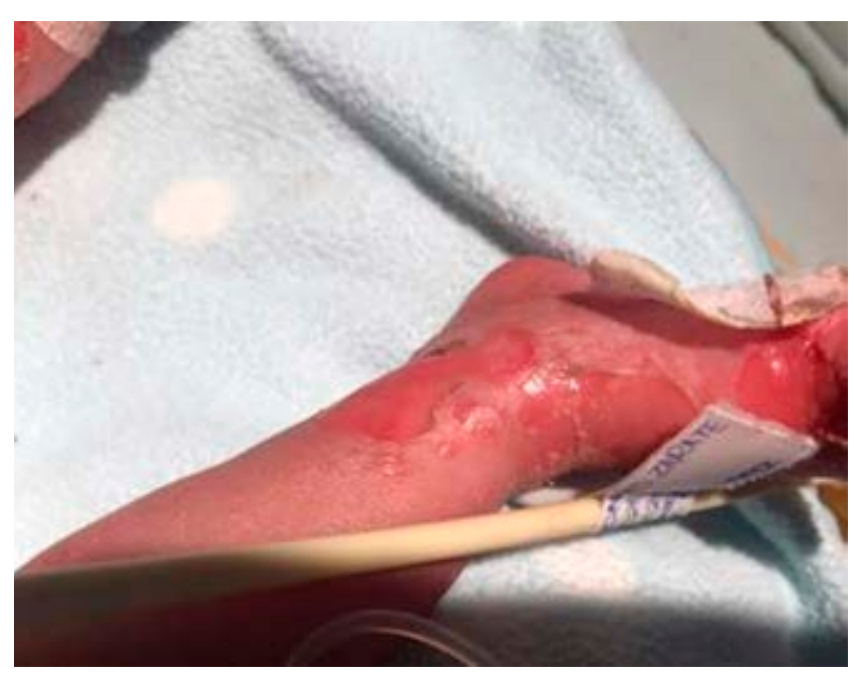

Figura 3: Desprendimiento de ampollas en tobillos y dorso del pie.

ampollas frágiles de contenido seroso asociado con sitios de trauma, de difícil reepitelización, y posteriormente en sitios de flexión como tobillos y muñeca, afectando mucosa oral (Figuras 1 a 3). Por tal motivo se practicó un drenaje estéril con aguja fina y fomentos de baño coloide. A los 14 días de vida, las lesiones se encontraron con fondo eritematoso con algunas ámpulas de contenido mielicérico, sospechándose de síndrome de piel escaldada, por lo que se inició cefotaxima y vancomicina. Posteriormente, al persistir con intolerancia a la vía enteral asociada con vómitos, se ejecutó una laparotomía exploradora con resección de membrana pilórica y piloroplastia. Se inició un drenaje de sonda orogástrica en pozos de café, se efectuó un gastrograma, en donde se observó gastromegalia sin observarse paso del medio de contraste, por lo que se operó una laparotomía exploradora con gastroyeyunoa- nastomosis, presentando datos de respuesta inflamatoria sistémica escalando antibioticoterapia a piperacilina con tazobactam. Además, se evidenció insuficiencia respiratoria, requiriendo por ello ventilación mecánica invasiva. Se prosiguió por inmunología, reportando niveles de inmunoglobulinas séricas con IgA $<0.25 \mathrm{mg} / \mathrm{dL}$, IgG 3.2 $\mathrm{mg} / \mathrm{dL}$, IgM $<0.25 \mathrm{mg} / \mathrm{dL}$, IgE $<0.16 \mathrm{mg} / \mathrm{dL}$, por lo que se indicó gammaglobulina a $2 \mathrm{~g} / \mathrm{kg} /$ dosis. Luego se realizó biopsia de piel por sacabocados, reportando dermatitis de interfaz vacuolar pauciinflamatoria linfocitaria perivascular superficial, compatible con necrólisis epidérmica tóxica (Figura 4). Finalmente, presentó datos de choque séptico secundario a Candida albicans, con deterioro respiratorio y hemodinámico, por lo que desafortunadamente falleció.

\section{DISCUSIÓN}

Los trastornos cutáneos ampollosos en los neonatos son poco frecuentes y es muy difícil hacer la diferenciación clínica o establecer un patrón determinado, por lo que el diagnóstico definitivo se establece por biopsia; dentro de este grupo se incluyen los autoinmunes como penfigoide, dermatosis lineal por IgA, epidermólisis ampollosa. Otras causas son el síndrome de piel escaldada y farmacodermias. Se describe un caso de sobreposición de SSJ/NET durante el periodo neonatal, identificándose, reportados en la literatura hasta el momento, cuatro pacientes en esta etapa de la vida, asociados con sepsis por Staphylococcus coagulasa negativos, ${ }^{7}$ Klebsiella pneumoniae ${ }^{8}$ y Escherichia coli. ${ }^{9}$ Así como un caso de NET asociado con fluconazol. ${ }^{10}$ Posiblemente la asociación con sepsis en los casos de NET en la etapa mencionada podría insinuar una asociación causal diferenciándola de la NET en niños mayores o adultos en donde se liga principalmente a medicamentos. A la par que consecuencia del estado hipermetabólico con
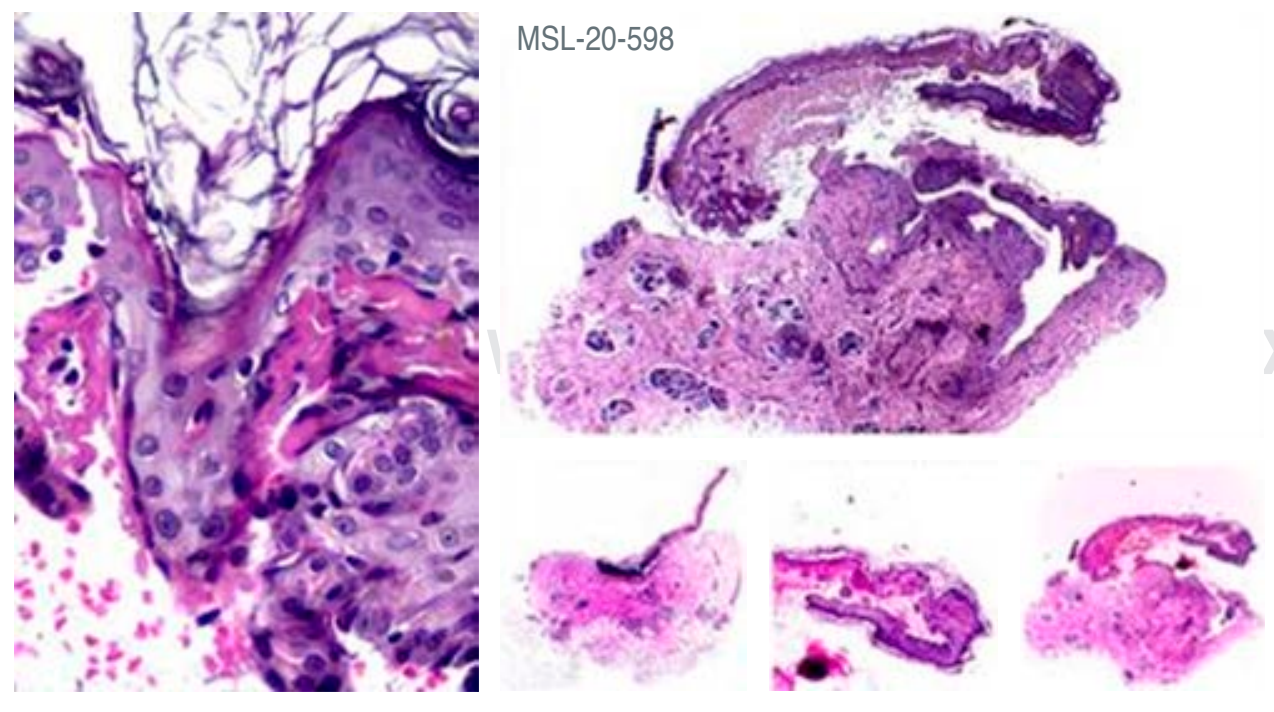

Figura 4:

Dermatitis de interfaz vacuolar pauciinflamatoria linfocitaria perivascular superficial. 
hipoalbuminemia e hipogammaglobulinemia asociado con la falla de la función protectora de la epidermis, se genera el riesgo de desarrollar sepsis, que es la primera causa de muerte al igual que sucedió en el paciente comentado, sin embargo, no es posible descartar que se trate de un error innato de la inmunidad en donde todos los isotipos de inmunoglobulinas están disminuidos como inmunodeficiencias combinadas, agammaglobulinemia ligada al $X$ y una nueva inmunodeficiencia autosómica recesiva caracterizada por la ausencia de células B, agammaglobulinemia y formación de ampollas en la unión dermoepidérmica de inicio temprano descrita como mutaciones hipomórficas de SLC39A7 que codifica el transportador de zinc del retículo endoplásmico al citoplasma ZIP7. ${ }^{11}$

\section{CONCLUSIÓN}

La superposición SSJ/NET es extremadamente rara durante el estadio neonatal. Es de vital importancia realizar un diagnóstico definitivo a través de la toma de biopsia para poder otorgar un tratamiento oportuno, debido a la alta mortalidad registrada en este grupo de edad. Si bien en niños y adultos la causa fundamental son los fármacos, en las publicaciones de los casos transcurridos durante dicha etapa, el padecimiento se asocia principalmente a infecciones que conllevan una elevada mortalidad. Se necesitan más estudios a fin de determinar las estrategias de tratamiento más efectivas.

\section{REFERENCIAS}

1. Tristani-Firouzi P, Petersen MJ, Saffle JR, Morris SE, Zone JJ. Treatment of toxic epidermal necrolysis with intravenous immunoglobulin in children. J Am Acad Dermatol. 2002; 47 (4): 548-552.

2. Frey N, Jossi J, Bodmer M, Bircher A, Jick SS, Meier CR et al. The epidemiology of Stevens-Johnson syndrome and toxic epidermal necrolysis in the UK. J Invest Dermatol. 2017; 137 (6): 1240-1247.

3. McPherson T, Exton LS, Biswas S, Creamer D, Dziewulski P, Newell $L$ et al. British Association of Dermatologists' guidelines for the management of Stevens-Johnson syndrome/toxic epidermal necrolysis in children and young people, 2018. Br J Dermatol. 2019; 181 (1): 37-54.

4. Lee HY, Chung WH. Toxic epidermal necrolysis: the year in review. Curr Opin Allergy Clin Immunol. 2013; 13 (4): 330-336.

5. Demoly P, Adkinson NF, Brockow K, Castells M, Chiriac AM, Greenberger PA et al. International Consensus on drug allergy. Allergy. 2014; 69 (4): 420-437.

6. Corrick F, Anand G. Question 2: Would systemic steroids be useful in the management of Stevens-Johnson syndrome? Arch Dis Child. 2013; 98 (10): 828-830.

7. Lohmeier K, Megahed M, Schulte KW, Stannigel H, Mayatepek E, Schroten $\mathrm{H}$. Toxic epidermal necrolysis in a premature infant of 27 weeks' gestational age. Br J Dermatol. 2005; 152 (1): 150-151.

8. de Groot R, Oranje AP, Vuzevski VD, Mettau JW. Toxic epidermal necrolysis probably due to Klebsiella pneumoniae sepsis. Dermatologica. 1984; 169 (2): 88-90.

9. Scully MC, Frieden IJ. Toxic epidermal necrolysis in early infancy. J Am Acad Dermatol. 1992; 27 (2 Pt 2): 340-344.

10. Islam S, Singer M, Kulhanjian JA. Toxic epidermal necrolysis in a neonate receiving fluconazole. J Perinatol. 2014; 34 (10): 792-794.

11. Anzilotti C, Swan DJ, Boisson B, Deobagkar-Lele M, Oliveira C, Chabosseau $\mathrm{P}$ et al. An essential role for the $\mathrm{Zn} 2+$ transporter ZIP7 in B cell development. Nat Immunol. 2019; 20 (3): 350-361. 